\title{
Is mitral stenosis associated with gastrointestinal bleeding: A twist on Heyde's Syndrome
}

\author{
Nguyen James ${ }^{1 *}$, Vyas $\mathrm{N}^{1}$, Alkhawam $\mathrm{H}^{1}$, Sogomonian $\mathrm{R}^{1}$, Saker $\mathrm{E}^{2}$, Ahmad $\mathrm{S}^{1}$ and Rubinstein $\mathrm{D}^{1}$ \\ ${ }^{1}$ Icahn School of Medicine, Mount Sinai (Elmhurst), Department of Cardiology, 79-01 Broadway, Queens, NY, USA \\ ${ }^{2}$ St. George's University School of Medicine, St. George's, Grenada, USA
}

\begin{abstract} would mitral stenosis (MS) cause a similar presentation? concomitant AS were excluded.

\section{Introduction}

Heyde's syndrome is the link between Aortic stenosis(AS) and angiodysplasia leading to Gastrointestinal (GI) bleed. It was first noted by Dr. Edward C. Heyde back in 1958 as GI bleeding of idiopathic sources was significantly more prevalent among patients with AS [1]. There have been multiple theories behind Heyde's syndrome; however, it is now believed to be caused by a depletion of Von Willebrand factor (vWF), similar to Von Willebrand disease type IIA (vWD-2A). Bleeding is thought to occur due to angiodysplasia colonic vascular ectasias that is unable to clot due to destruction and depletion of vWF; this occurs as vWF flows through the narrowed valve and cleaved by metalloproteinase [2]. This association is further validated by the cessation of GI bleed after aortic valve replacement. The question posed is, if AS can lead to GI bleeding through loss of vWF or other mechanisms, would MS cause a similar presentation?
\end{abstract}

Introduction: Heyde's syndrome is the link between Aortic stenosis (AS) and angiodysplasia leading to Gastrointestinal (GI) bleed. There have been multiple cases and theories leading to this phenomenon, including the loss of von Willebrand factor (vWF) through shear factor of a stenotic valve. This association is further validated by the cessation of GI bleed after aortic valve replacement. The question posed is, if AS can lead to GI bleeding through loss of vWF or other mechanisms,

Methods: A single center, retrospective chart analysis was done on patients, age 18 and over, with evidence of MS on echocardiogram for any signs of GI bleeding. The outpatient clinic notes and admission notes, along with colonoscopies to detect the presence of angiodysplasia were reviewed for GI bleeding. Patient's with

Results: MS group were 35\% males and 65\% female. Average age was 61 years old. Approximately $4 \%$ had MS secondary to rheumatic heart disease and $45 \%$ due to calcified annulus. Of 162 patients with MS, 7 (4.3\%) patients had evidence of gastrointestinal bleed versus 16 (10\%) of non-MS group (p=0.06). Patients with MS and GI bleed were found to have arteriovenous malformation (AVM) (35\%), gastric or duodenal ulcer (35\%), colon cancer (3\%) and diverticulitis (37\%).

Conclusion: MS does not have an increase incidence of GI bleeding when compared to the control group, though the $\mathrm{P}$ value was not statistically significant. $\mathrm{WF}$ is thought to be decreased because of increased shear force through a stenotic valve. Flow through the stenotic mitral valve is orders of magnitude lower than the flow through a stenotic aortic valve given the force of contractility in the atria compared to the ventricle, therefore is unlikely to cause decrease in vWF to lead to GI bleeds.

\section{Methods}

\section{Study design}

A single-centered, retrospective chart analysis was performed between 2004 and 2014 of patients who had evidence of MS, and matched with non-MS group. This study was conducted at a major hospital center in one of the most diverse communities in the United States, providing a culturally and epidemiologically significant advantage. An approved retrospective chart analysis using QuadraMed Computerized Patient Record (QCPR) and accessed with data-input and calculations formulated in a computerized software. This study has been approved by Institutional Review Board of the Mount Sinai School of Medicine (HS\#: IRB-16-00393).

\section{Selection criteria}

One hundred and sixty-two patients had evidence of MS based on 2D echocardiogram. Patient's with concomitant AS were excluded. Among these patients, the chart was reviewed for admissions suspected of upper or lower GI bleed with signs of anemia, and had either Esophagogastroduodenoscopy (EGD), Colonoscopy or both. The underlying cause of MS was identified, and a random matched control group without MS was selected (Table 1). For all statistical analysis, the results were considered significant when two-tailed p-values were $<0.05$. The distributions of age, sex and baseline co-morbidities were compared between the two cohorts.

\section{Results}

MS group were 35\% males and 65\% female with average age of 61 years old. Approximately $4 \%$ had MS secondary to rheumatic heart disease and $45 \%$ due to calcified annulus. Of 162 patients with MS, 7 (4.3\%) patients had evidence of gastrointestinal bleed versus $16(10 \%)$

Correspondence to: Nguyen James, Icahn School of Medicine, Mount Sinai (Elmhurst), Department of Medicine, 79-01 Broadway, Queens, NY, USA, E-mail: james.nguyen@mssm.edu

Received: March 17, 2017; Accepted: April 18, 2017; Published: April 21, 2017 
Table 1. Statistical analysis.

\begin{tabular}{|l|c|c|}
\hline & MS group n=162 & Non- MS group n=162 \\
\hline Average age & 61 years old & 63 years old \\
\hline Gender M/F & $57 / 105$ & $55 / 107$ \\
\hline Ethnicity & Hispanic $60 \%$ & Hispanic $58 \%$ \\
\hline History of Rheumatic heart disease & $4 \%$ & $3.5 \%$ \\
\hline Calcified annulus & $45 \%$ & $43 \%$ \\
\hline Evidence of GI bleeding & 7 & 16 \\
\hline
\end{tabular}

of non-MS group ( $\mathrm{p}=0.06)$. The causes of GI bleed in patients with MS were as follows: arteriovenous malformation (AVM) (35\%), gastric or duodenal ulcer $(35 \%)$, colon cancer $(3 \%)$ and diverticulitis $(37 \%)$.

\section{Discussion}

\section{MS is caused by five main conditions}

Rheumatic disease, congenital abnormalities, active infective endocarditis, metabolic or enzymatic abnormalities such as gout or whipples massive annular calcification [3] The severity of MS can be broken down into: mild with gradient of $8 \mathrm{~mm} \mathrm{Hg}$, moderate with gradient 8 to $12 \mathrm{~mm} \mathrm{Hg}$ and severe with gradient $12 \mathrm{~mm} \mathrm{Hg}$ or higher [4]. According to the data we gathered, there is no association between MS and angiodysplasia or GI bleeding. The accepted pathogenesis of Heyde's syndrome is acquired vWF deficiency due to shearing. Shear rate is directly proportional to the blood flow velocity and inversely proportional to vessel diameter. Under normal physiologic situations, the highest wall shear rates occur in the arterioles 4,000 seconds $^{-1}$, which enhance platelet/vWF interactions $[5,6]$.

The vWF abnormality is related to the severity of stenosis [7]. Severe AS can produce shear rates as high as 10,000 seconds $^{-1}$, which induces a conformational change to the high molecular weight (HMW) vWF multimers, exposing the bond between amino acids Tyr842 and Met843, leading to proteolysis by ADAMTS13, an important disintegrin and metalloprotease that cleaves vWF at the A2 domain [59]. This effectively decreases the number of HMW multimers leading to an acquired vWD-2A and impairing hemostasis at angiodysplasic vessels, causing to a tendency to bleed, as HMW multimers are the most platelet reactive, and aide clotting $[6,9,10]$. In MS, the velocity increases from a normal value of less than $1 \mathrm{~m} / \mathrm{sec}$ to a value greater than $1.5 \mathrm{~m} / \mathrm{sec}$ [11] and even with significant decrease in mitral valve area in patients, peak and mean trans mitral gradient do not significantly change [12]. In addition, the atrial contraction or atrial kick accounts for roughly $10 \%$ of ventricular filling, and up to $24 \%$ across a stenotic mitral valve, depending on degree of stenosis and mitral valve area, which fluctuates with flow $[13,14]$. When considering force of contraction of the atrium to the force of contraction of the ventricle, where normal ejection fraction is $65 \%$, there is large discrepancy in force and velocity generated between the two chambers, showing a clear difference in shear force. Finally, atrial fibrillation occurs in $40-75 \%$ of patients with MS, [15] which effectively removes the force produced by the atrium, further effectively decreasing shear rate. Therefore, shear in MS is negligible when compared to AS, and would not cause the conformational change and destruction that would lead to an acquired vWF deficiency. In a study of 500 operatively excised mitral valves, $45 \%$ of the valves had concomitant stenotic and regurgitation features [3], which would prompt a patient to become further symptomatic and receive mitral valve replacement. In patients with GI bleed and AS, shear stress was significantly reduced after valve replacement [16] and further studies showed that aortic valve replacement stopped bleeding in as high as $93 \%$ of patients $[10,17,18]$ and thought to be the solution for this syndrome. Therefore, even if MS had enough shear force to cause an acquired vWF deficiency, a fair number of patients would have had the required treatment early on and not experience GI bleed, unless from anticoagulation for valve replacement.

\section{Conclusion}

Finally, with MS, there is a risk of left atrial thrombus due to increased systemic levels of byproducts from the coagulation cascade. Studies have shown that there are increased prothrombin fragments in the left atrium $[19,20]$ and this hypercoagulable state is likely due to sluggish blood flow and low shear rate conditions. Therefore, MS may actually have less incidence of bleeding which is what our data showed, though the data set is small and statistically not significant. High shear rates, which also occur pathologically when blood flows rapidly through larger but stenotic vessels, MS does not have an increase incidence of GI bleeding when compared to the control group, though the $\mathrm{P}$ value was not statistically significant and may actually have less incidence given a possible hypercoagulable state from having MS. vWF is thought to be decreased because of increased shear force through a stenotic valve, leading to degradation; however, flow through the stenotic mitral valve is orders of magnitude lower than the flow through a stenotic aortic valve given the force of contractility in the atria, or lack of, in atrial fibrillation, as compared to the force of contraction in the ventricle. Therefore, is unlikely to cause decrease in vWF that leads to GI bleeding from angiodysplastic vessels.

\section{References}

1. Heyde EC (1958) “Gastrointestinal bleeding in AS.” N Engl J Med 259: 196.

2. Greenstein RJ, McElhinney AJ, Reuben D, Greenstein AJ (1986) Colonic vascular ectasias and AS: coincidence or causal relationship?. Am J Surg 151: 347-351. [Crossref]

3. Waller BF, Howard J, Fess S (1994) Pathology of mitral valve stenosis and pure mitral regurgitation-Part I. Clin Cardiol 17: 330-336. [Crossref]

4. Batur P, Stewart WJ, Isaacson JH (2003) Increased prevalence of AS in patients with arteriovenous malformations of the gastrointestinal tract in Heyde syndrome. Arch Intern Med 163: 1821-1824. [Crossref]

5. Warkentin TE, Moore JC, Anand SS, Lonn EM, Morgan DG (2003) Gastrointestinal bleeding, angiodysplasia, cardiovascular disease, and acquired von Willebrand syndrome. Transfus Med Rev 17: 272-286. [Crossref]

6. Warkentin TE, Moore JC, Morgan DG (1992) Aortic stenosis and bleeding gastrointestinal angiodysplasia: is acquired von Willebrand's disease the link? Lancet 340: 35-37. [Crossref]

7. Vincentelli A, Susen S, Le Tourneau T, Six I, Fabre O, et al. (2003) Acquired von Willebrand syndrome in aortic stenosis. $N$ Engl J Med 349: 343-349. [Crossref]

8. Morishima A, Marui A, Shimamoto T, Saji Y, Tambara K, et al. (2007) Successful aortic valve replacement for Heyde syndrome with confirmed hematologic recovery. Ann Thorac Surg 83: 287-288. [Crossref]

9. Pareti FI, Lattuada A, Bressi C, et al. (2000) Proteolysis of von Willebrand factor and shear stress-induced platelet aggregation in patients with aortic valve stenosis. Circulation 102: 1290-1295.

10. Thompson JL 3rd, Schaff HV, Dearani JA, Park SJ, Sundt TM 3rd, et al. (2012) Risk of recurrent gastrointestinal Bleeding after Aortic valve replacement in patients with Heyde Syndrome. J Thorac Cardiovasc Surg 144: 112-116. [Crossref]

11. Baumgartner, Helmut, et al. (2008) Echocardiographic assessment of valve stenosis EAE/ASE recommendations for clinical practice. Eur Heart J-Cardiovasc Imag.

12. Sagie A, Freitas N, Padial LR, Leavitt M, Morris E, et al. (1996) Doppler echocardiographic assessment of long-term progression of mitral stenosis in 103 patients: valve area and right heart disease. J Am Coll Cardiol 28: 472-479. [Crossref]

13. Mohan JC, Patel AR, Passey R, Gupta D, Kumar M, et al. (2002) Is the mitral valve area flow-dependent in MS? A dobutamine stress echocardiographic study. J Am Coll Cardiol 40: 1809-1815. [Crossref] 
14. Meisner JS, Keren G, Pajaro OE, Mani A, Strom JA, et al. (1991) Atrial contribution to ventricular filling in mitral stenosis. Circulation 84: 1469-1480. [Crossref]

15. Chandrashekhar Y, Westaby S, Narula J (2009) Mitral stenosis. Lancet 374: 1271-1283. [Crossref]

16. Gostout CJ (1995) Angiodysplasia and aortic valve disease: let's close the book on this association. Gastrointest Endosc 42: 491-493. [Crossref]

17. Islam S, Islam E, Cevik C, Attaya H, Otahbachi M, et al. (2012) Aortic stenosis and angiodysplastic gastrointestinal bleeding: Heyde's disease. Heart Lung 41: 90-94. [Crossref]
18. Massyn MW, Khan SA (2009) Heyde syndrome: a common diagnosis in older patients with severe aortic stenosis. Age Ageing 38: 267-270. [Crossref]

19. Atak R, Yetkin E, Yetkin O, Ayaz S, Ileri M, et al. (2003) Increased systemic and regional coagulation activity in patients with MS and sinus rhythm. Angiology 54: 593597. [Crossref]

20. Gur C, Lalazar G, Raphaeli G, Gilon D, Ben-Chetrit E (2006) Mitral stenosis presenting with acute hearing loss. PLoS Med 3: e233. [Crossref]

Copyright: @2017 James N. This is an open-access article distributed under the terms of the Creative Commons Attribution License, which permits unrestricted use, distribution, and reproduction in any medium, provided the original author and source are credited. 